\title{
XQED: THE NEXT GENERATION OF WET LIGHT CURVE REDUCTION AND ANALYSIS SOFTWARE
}

\author{
R. L. Riddle \\ Dept. of Physics and Astronomy, Iowa State University, Ames, IA 50011, \\ U.S.A.
}

Received January 13, 2003

\begin{abstract}
For many years, the Whole Earth Telescope has used the QED software package, created by R. E. Nather, to reduce the data gathered from the WET standard, PMT based photometers. While essential for reducing this data, QED alone is not a sufficient package for reducing CCD photometry data, which is becoming a larger fraction of WET data. In addition, QED requires DOS, while many astronomers, and WET HQ, do everything in a different environment (usually Unix based). A new version of the data reduction software will allow the WET to continue to operate with future CCD photometers and systems, and to reduce archival photometry data with new computer systems. Here a software package is described which will satisfy these new needs.
\end{abstract}

Key words: techniques: photometric - methods: data analysis

\section{INTRODUCTION}

QED was developed to reduce photometric data obtained using Quilt 9, the software that controls the WET standard, PMT based photometer (Kleinman, Nather \& Phillips 1996). QED was based upon an earlier data reduction program called DRED, written by Butler Hine at U. Texas. As the name may imply, DRED was not the most user friendly software package around, so Ed Nather improved on it by creating QED, which uses the Quilt 9 keystrokes and display (to prevent brain confusion/damage among users), and expands on DRED to include useful elements such as an undo function, as well as every necessary element of the data reduction process. QED also does not crash at an inappropriate keystroke, and limits the user in processing order and variable values in an attempt to guarantee the quality of the output.

While QED has been a critical reduction tool for the WET pho- 
tometer data, it is becoming less so as more WET sites move to CCD photometry. During XCov22, about half of the twenty one participating sites used CCDs to gather their data, each with their own format for extracted photometry data. Data from only one site could be reformatted to the Quilt 9 format so that QED could be used to reduce its data; two others were submitted in Quilt 9 format, but QED only allowed limited capability in reducing the data.

A much larger issue is that QED is a DOS based program; it will only run in a Unix environment (such as the WET HQ) in emulation, and emulation almost always brings about compatibility issues. In the case of HQ, only the VMWare DOS emulation package (VMWare) has proved a viable solution; other emulators either do not display the graphics properly, or they have problems with keystroke identification or other fatal issues. VMWare is a commercial package, and HQ can only run it under license on two computers, limiting the reduction process.

All of these issues will only become larger in the future, so a solution to the data reduction system is required now. Anticipating this dilema, Ed Nather suggested to the WET collaboration that someone should undertake the creation of a new version of QED, ostensibly to run under Xwindows and be portable to many flavors of Unix. Fortunately, I had already started to investigate the creation of an Xwindows version of QED.

\section{SO, WHY DO WE WANT THE NEW VERSION?}

First and foremost, a new version of QED is required that can be updated, expanded and able to run on future hardware and operating systems. Currently, the Unix environment, and Linux in particular, is ubiquitous in astronomical institutions. Developing XQED under the Unix environment lessens the possibility that the architecture may go "out of style", or that it may be replaced with a new architecture that is no longer compatible.

As the WET moves to CCDs, we will require software that can swiftly reduce the data. It is likely that there will be changes in how the WET data reduction, or CCD photometry in general, is done within the next twenty years; if XQED is flexible enough, it may be adapted to these changes, while QED is unable to adapt to CCD data now without shoehorning the data into a particular, inflexible format. XQED is being created with CCD data in mind, so its data format will be more flexible than the Quilt 9 format, to allow different CCD photometry extraction algorithms to adjust their output 
to be compatible. All of the reduction and analysis tools available to QED will be incorporated into XQED, and there will be ample opportunity to expand those capabilities in the future. The development of XQED in the Unix/Xwindows environment may also allow it to become useful to astronomers outside the WET collaboration.

XQED will, in all likelihood, be released under a GNU Public License, which will allow others to contribute their own ideas and tools to the software. At the moment, many in the WET collaboration use their own software packages for further data reduction; XQED is intended to be the first in a suite of software that everyone can use and contribute to, hopefully bringing the best aspects of the separate packages into a powerful whole.

\section{THE NEW KID IN TOWN}

The process of creating a new version of QED seems straightforward enough: create an interface to display the light curve and interact with it, and then include the QED data reduction algorithms. The difficulty, however, is that the every bit of the display must be created anew, as none of it is transportable from the DOS display system QED uses. For this reason, and because QED is still required for most WET data, no one has attempted to create a new version of QED. It is a large undertaking; just reproducing QED in Xwindows is difficult, and expanding its usefulness is even harder. All of this is a testament to the hard work of Ed Nather in developing and maintaining QED.

\subsection{Design considerations}

The new interface should use the usual Xwindows paradigm of menus, mouse and "point and click" interaction (which in itself is a large change from QED); this drives many other changes in the software. For example, in QED it is impossible to see the data plot simultaneously with any other information (such as help screens or the reduction $\log$ ). The new software can be designed to support multiple windows, so multiple bits of information can be continuously available. Many other new, useful functions can be added to the software, but each item increases the complexity at the same time. For the initial version, I am attempting to leave in pathways to future functions while creating just the basics of the data reduction system that we need.

Once the interface is created, then it should be relatively simple to take the operational aspects of QED and move them into the new 


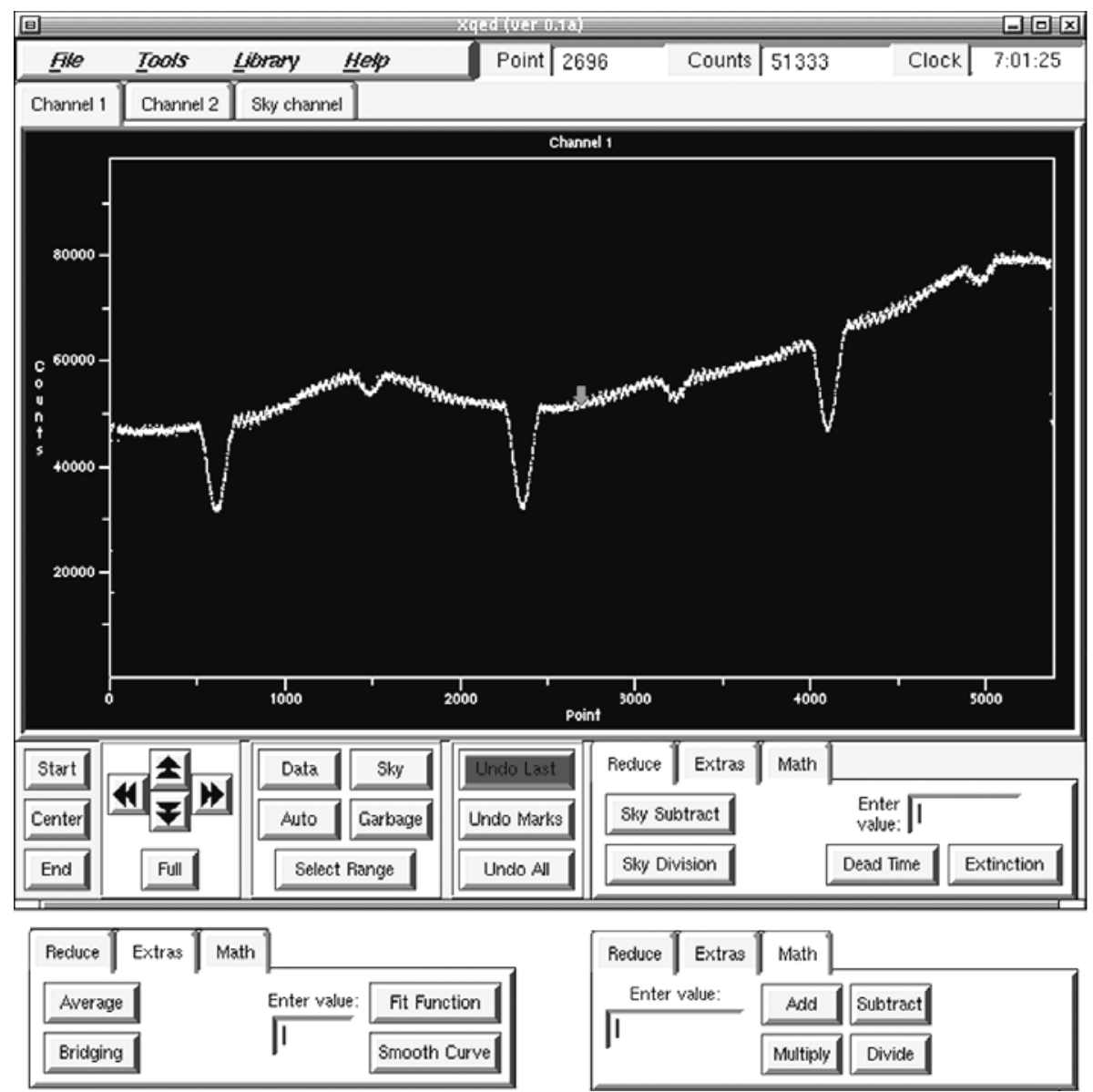

Fig. 1. The main reduction window of the XQED package. The two tab windows at the bottom are the other functions included in the lower right tabs.

package. All of these algorithms will likely require some modification in order to interact with the Xwindows portion of the software, so the porting process will likely be more complicated. In addition, some elements may require revision in and of themselves, such as an update to the calculation of BJED. Creating the interface itself should be the more complicated aspect of the development, however.

\subsection{The software}

First of all, everything in this section may turn out to be hopelessly optimistic, completely wrong or outdated by the time XQED is released. So far, programming XQED seems as much an exercise in creating a new package as it is in teaching me how little I 
know about software development. However, unless some huge show stopping issue appears, it seems that what follows will be how the software eventually develops.

XQED will be based on the Xforms library (Xforms library); Xforms has been released under the GNU GPL license, and is an excellent Xwindows graphical development environment. It also is compatible with almost any X11 system out there, requiring only a recompile; other Xwindows environments did not seem nearly as straightforward for development when this project was started. In addition, Xforms has very understandable output code (in C) that can be connected to other algorithms, while other development environments did not seem nearly as easy to dissect and connect. The newest version is currently available at the Xforms download site mentioned in the references; it will be required to download and install the library in order to run XQED. There is also a mailing list that discusses the library development in the references.

The XQED environment will be composed of several separate windows, each of which encompasses the functions of the original while expanding them in useful ways. The current main interaction window is displayed in Figure 1. On first glance, this looks similar to QED, with a plot of the light curve in place, and information about the light curve point in the upper right corner.

The keyboard interaction will be similar to QED, but each light curve is in a separate plot, and brought up by clicking on the tabs at the top of the plot window, instead of only having two overlaid. This is to accommodate the inclusion of many channels of data; where QED handles three channels, and displays two, XQED handles 12 (at the moment). Unfortunately, there is no easy way to distinguish between that many channels, or display them in a cohesive way, in a single plot. There will be a "viewing" window that can be used to select channels to overlay, but that has not been programmed yet.

The real power of the new reduction window is the use of color to differentiate between different parts of the plot. The marker for the current point is a bright green arrow, sky points are blue, and garbage data points are red. A range of points can be selected, and they will display differently as well. In addition, this display responds to mouse interaction; at the moment, this is limited to moving the curve around with mouse clicks, but will expand to include a menu (for movement or changing the state of a point), and hopefully range selection. The goal is to create a data reduction that is truly point and click, which will hopefully simplify and enhance the reduction process and quicken the learning curve. 

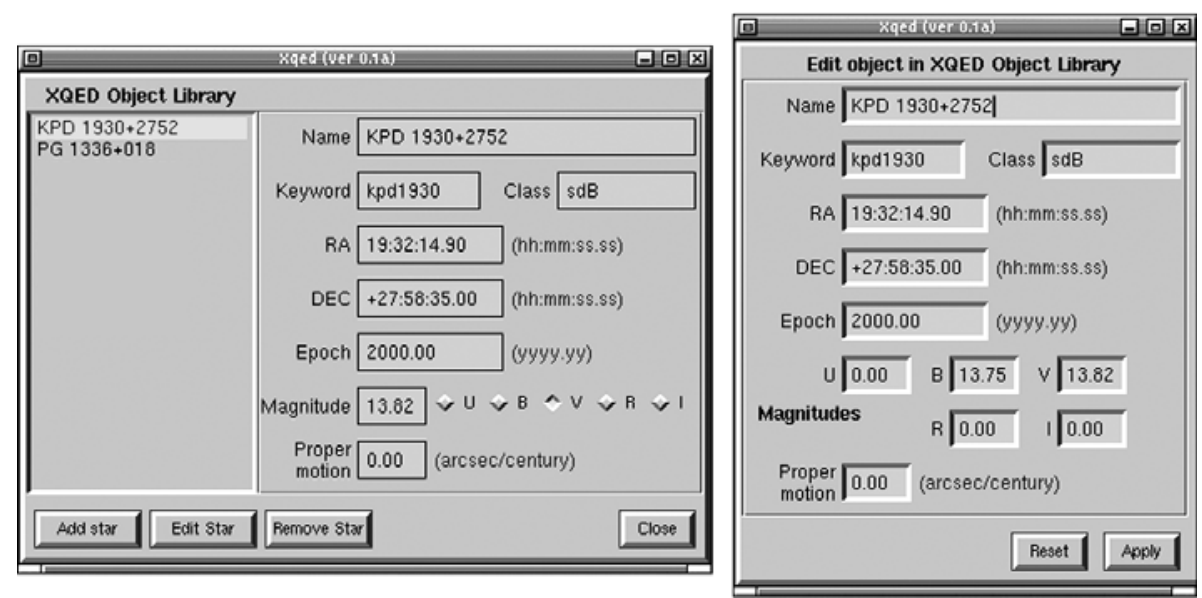

Fig. 2. Library interaction windows. The left window is the library list; selecting from the list displays object information. It is also possible to Add an object, Remove an object from the list or Edit an object (shown to the right).

In Fig. 1, the top right portion of the window includes the usual information about the current point, with the point selected (color coded for the point state), the counts at the point and the clock time for that point during the observation. The menu bar on the top left is separated into four menus:

File This menu contains options for opening files, saving the current file and exiting the program. It also opens the Preferences, which will allow setting items like point size, key strokes and other parameters.

Tools Since the reduction window in XQED does not allow different light curves to be overplotted, there will be a separate View window that will allow that function. Different light curves can be examined, with either each in separate windows or all overlaid on a single plot. This menu will also allow output of the final data plot, and likely contain a Fourier Transform plotting function, for examination of how certain reduction steps affect the output FT as the reduction is done (in other words, "realtime"). In addition to the plotting tools, other items related to the reduction process will be found here.

Library XQED will include several library files. Each of these will be editable by the user, and include such things as observatories, objects, and photometers. The libraries will be a standard format used by other WET tools, and will periodically be up- 


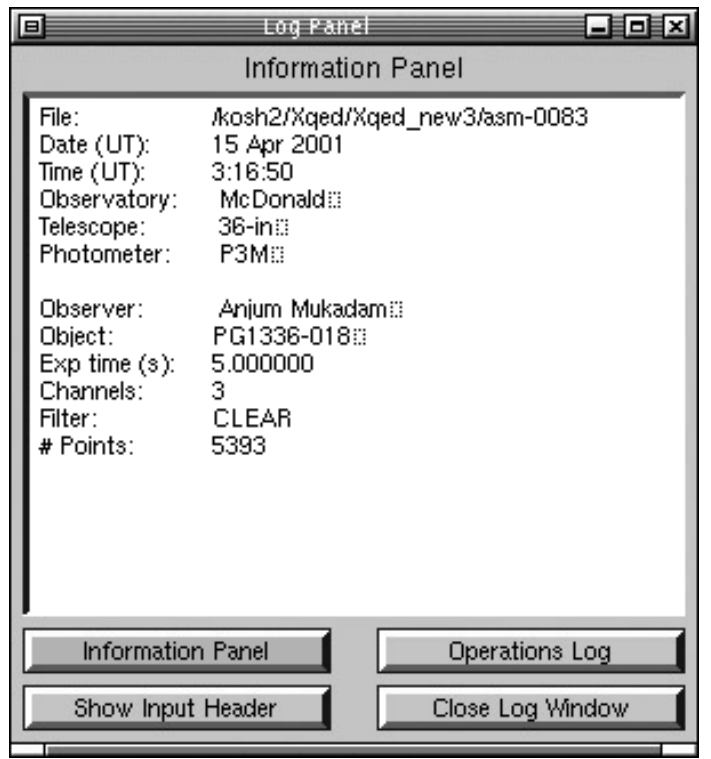

Fig. 3. The XQED log window.

dated with new information. The libraries will be editable; Figure 2 shows the Object library interaction window (with a test list of objects), and a window that allows editing the values for one library entry. There will also be a "Reset Library to default" switch, just in case (though the user will lose all added information in that case).

Help Integrated help will be included with the package, both through popup tags on interface items and in an online manual.

Many of these elements will be developed later, and it's very possible that the menus may be shuffled as functionalities change and/or other items are required to be added. The features of the embedded, editable libraries, the active plots and the included FT functions will be useful improvements over QED. Also, individual preference settings (so people don't need to argue about which direction the left arrow should move the cursor) gives each user an opportunity to tune their reduction to their liking. These enhancements will likely take a while to appear, but they are also a major reason why I undertook this project.

The bottom portion of the window includes the rest of the elements necessary to reduce the data; most of these will be repeated via keyboard control, though some of the input parameters will, by necessity, require entry into the interface. On the left are buttons 
to move to the start, center or end of the light curve; mouse and/or keyboard control is used to move around otherwise, so a "movement keypad" is redundant. The next group of buttons control magnification (or scaling) of the plot, with a button for a full scale plot. Data marking buttons are included as well, though this will likely be superfluous. The undo function buttons are included, but only the "Undo marks" for points is in place. The other undo functions will be created eventually, but the software must do something before it can undo it.

The tab folders at the bottom right include all the advanced reduction tools; each tab includes different elements, organized in complementary groups. The Reduction tab, includes the Sky subtraction, Dead time and Extinction functions, all of which are necessary for any data reduction. The Extras tab includes smoothing, fitting and other functions that come in handy but that aren't always necessary. A Math panel is included as well for those useful operations. The operations in these panels may not be interfaced to the keyboard, especially those that require input; this part of the interface is the newest, and still under development. Extra panels can be addd if functionality beyond those above are developed.

The log window (Figure 3) will include a list of the input header data, the reduction parameters being used (from the library), and the list of operations in the data reduction process. The reduction parameters will be editable, so that errors in the data header can be removed and additional information can be added.

\section{CONCLUSION}

There are many other planned features of XQED. It already includes error checking, and this will be expanded to give comprehensive feedback when something goes amiss. As with QED, elements of the software will attempt to keep the user from doing extremely boneheaded things, but allow the flexibility to do all the useful items required. There is also hope that there will be a comprehensive plotting function, for output of the data reduction, and an included, live Fourier Transform, which will show how the FT changes as different operations are done to the data. There will also be preferences for the keymapping, colors of the plot and other parts of the software, though this will be lower priority for a while. XQED will take a while to develop, so many features will not appear at first; it is hoped that they will appear soon, and that contributions of ideas from the community will help to expand the capabilities of the software as it 
develops.

The new XQED package will, most likely, require an extended test period to ensure that it does, in fact, reduce photometry data appropriately. This will initially be internal at HQ, but we will allow the community to see and comment on the software once we feel that it actually works. At this writing, it is hoped that there will be some type of public release of XQED early in 2003. It is likely that this release will be only a bug test version, where the community will be asked to make sure that there are no errors in the data reduction algorithms. This will also be a chance for the community to comment on the usability of the software, although many of the planned "bells \& whistles" will not be in place.

The eventual goal is to have XQED used as the primary data reduction software for $\mathrm{HQ}$ at XCov23, with QED as a backup. Once we have a stable version that correctly reduces the data, then it will be declared version 1.0, and will likely be opened up to the community for development contributions. As always, software development takes longer than anticipated, so we shall have to wait and see how many hurdles appear in the development cycle.

ACKNOWLEDGMENTS. I would like to acknowledge the work of Ed Nather on the original QED. His discussions with me have been very helpful in guiding me to develop the new software...I only hope that I am one-tenth the programmer that he is, so that there is a chance this will be done one day. Thanks also to Steve Kawaler, and to many others who have made comments about QED that have helped me to see how to create a new version.

\section{REFERENCES}

Kleinman S. J., Nather R. E., Phillips T. 1996, PASP, 108, 356

VMWare, http://www.vmware.com

Xforms library, http://world.std.com/ xforms/

Xforms download, ftp://ncmir.ucsd.edu/pub/xforms/OpenSource

Xforms mailing list, http://bob.usuhs.mil/mailserv/xforms.html 